\title{
Effect of exercise on cancer-related fatigue: $A$ systematic review
}

\author{
STEFANIA CATALDI $\bowtie$, GIANPIERO GRECO, MARIO MAURO, FRANCESCO FISCHETTI \\ Department of Basic Medical Sciences, Neuroscience and Sense Organs, School of Medicine, University of \\ Study of Bari, Italy
}

\begin{abstract}
Cancer is the second leading cause of death in the world and cancer-related fatigue (CRF) is the most common disease in cancer patients that received radiotherapy, chemotherapy, hormone therapy and biotherapy. Many studies recommended physical activity and exercise to improve fatigue. This systematic review aims to provide a qualitative synthesis of Randomized Clinical Trials (RCTs) evaluating the effects of Aerobic, Resistance, Endurance and combined exercises on CRF versus control or different exercise group in cancer adult patients and survivors that did not receive palliative care. This systematic review is written and presented according to PRISMA protocols. Articles in the English language were collected using the PubMed and WoS databases from 2001 January 1st to 2019 September 1st. Only RCTs lasted 5 weeks or more were analysed of which CRF outcomes were examined. A total of 15 RCTs met our inclusion criteria. Different outcomes in CRF self-reports were found between Aerobic, Resistance, Endurance and Combined exercises. Findings suggest that exercise improves CRF, especially with aerobic or combined programs. The outcomes of trials could help exercise professionals to properly plan the sessions by dosing the volume and intensity. Nevertheless, more studies are needed to better understand the benefits of physical exercise on cancer patients.

Keywords: Aerobic; Resistance; Endurance; Combined exercise; Physical activity; Cancer.
\end{abstract}

\section{Cite this article as:}

Cataldi, S., Greco, G., Mauro, M., \& Fischetti, F. (2021). Effect of exercise on cancer-related fatigue: A systematic review. Journal of Human Sport and Exercise, 16(3), 476-492. https://doi.org/10.14198/jhse.2021.163.01

Corresponding author. Department of Basic Medical Sciences, Neuroscience and Sense Organs, School of Medicine, University of Study of Bari, Lungomare Starita 1, 70123 Bari (BA), Italy. https://orcid.org/0000-0002-5929-4766

E-mail: stefania cataldi@virgilio.it

Submitted for publication November 15, 2019.

Accepted for publication January 27, 2020.

Published July 01, 2021 (in press March 03, 2020).

JOURNAL OF HUMAN SPORT \& EXERCISE ISSN 1988-5202

(c) Faculty of Education. University of Alicante

doi:10.14198/jhse.2021.163.01 


\section{INTRODUCTION}

Cancer is a group of diseases characterized by the uncontrolled growth and spread of abnormal cells. If the spread is not controlled, it can result in death. It is the second leading cause of death in the world and its incidence is growing, with 21.7 million new yearly cases predicted worldwide by 2030 (Torre et al., 2015). Five states with the highest incidence are Denmark $(338 / 100,000)$, France $(325 / 100,000)$, Australia $(323 / 100,000)$, the USA $(318 / 100,000)$ and South Korea $(308 / 100,000)$ (Kessels et al., 2018). For example, the American Cancer Society (2019) estimated 1,762,450 of new cases for cancer in USA and about 371,000 in Italy during 2019 (AIOM, 2019). Cancer is caused by internal factors, such as inherited genetic mutations, hormones, and immune conditions and external factors, such as tobacco, infectious organisms, and an unhealthy diet; in 2012, USA registered about $5 \%$ of all cancers were caused by inactivity (Parkin et al., 2011). Furthermore, Cancer was estimated to have resulted in 208.3 million disability-adjusted-life-years in 2015 (Bernsten et al., 2017). One of these is cancer-related fatigue (CRF) (Akechi et al., 1999).

CRF is defined by the National Comprehensive Cancer Network (NCCN, 2003) as "a persistent, subjective sense of tiredness related to cancer or cancer treatment that interferes with usual functioning"; $70-100 \%$ of patients being treated for cancer are affected by CRF, which can be more distressing and disruptive to daily activities than the pain associated with the disease (Curt et al., 2000). It is a common disease in patients who received radiotherapy, chemotherapy, hormone therapy and biotherapy (Jacobsen et al., 1999; Robinson \& Posner, 1992; Sitzia \& Huggins, 1998; Stone et al., 2000). CRF was added to "International Classification of Disease" (Cella et al., 2001) and guidelines for its management were made (NCCN, 2003). Its effects may be incurred during treatments and they persist for many months or years (Bower et al., 2000; Broeckel et al., 1998). The effects of CRF also have deleterious influences on patients' and survivors' physical, mental, and emotional well-being (Cataldi, Latino, Greco, \& Fischetti, 2019; Hofman et al., 2007). Several factors have been identified in research as contributing to fatigue, but it is still unclear what is most influent (Ryan et al., 2007). Physiological and psychosocial factors play a part in the specific mechanisms involved in the development of CRF (Ahlberg et al., 2003; Fischetti et al., 2019); an import contribute was made by muscular metabolism (Brown et al., 2005; Forsyth et al., 1999; Isaksson et al., 2002; Lane et al., 1998; Lee et al., 2003; McCully et al., 1996). For assessment and management of CRF, different scales and questionnaire were made (Cleeland et al., 1999; Mendoza et al., 1999; Piper, 1997; Piper et al., 1989; Piper et al., 1998; Schwartz, 1998; Schwart \& Meek, 1999; Smets et al., 1995; Sutherland et al., 1999).

The NCCN's (2015) clinical practice guidelines recommend physical activity and exercise to improve fatigue, undergoing and/or after treatments. A recent meta-analysis showed that both exercise and psychological treatments have small effect sizes in CRF, whereas medication has no effects, with most effects of exercise during cancer therapy (Mustian et al., 2017). Despite the evidence of training's benefits, cancer survivors or patients report a significant decline in exercise after diagnosis, with less than $50 \%$ engaging in a beneficial exercises program (Humpel \& Iverson, 2007; Midtgaard et al., 2009). A Cochrane systematic review and meta-analysis by Cramp and Byron-Daniel (2012) examined the effects of exercises on CRF in patients who received palliative or non-palliative treatments. Another review of Kessels et al. (2018) exceeded this problem establishing the effects of exercises on CRF in a population not suffering from specific end-life-distress, but they used only MET to quantify the intensity of exercises. Also, we included RCT 3-armed.

This study aims to provide a systematic review of RCTs evaluating the effects of Aerobic, Resistance, Endurance and combined exercises on CRF versus control or different exercise group, confronted from baseline to one or more follow-ups, in cancer patients and survivors who were adult (18 years old or more) and who did not receive palliative care. Different self-report used for fatigue outcomes and exercise intensity 
was registered by common methods as percentage of $H R_{\max }$ (Cinke \& Thomas, 1981) or $\mathrm{HR}_{\mathrm{r}}$ (Cheng et al., 2002), RPE (Borg, 1970), METs (Mendes et al., 2018), 1RM (Delorme \& Watkins, 1948) and VO2peak (Noonan \& Dean, 2000).

\section{METHODS}

\section{Search strategy and information processing}

The systematic review is written and presented according to PRISMA protocols (Liberati et al., 2009). PubMed (a search engine for free access to the MEDLINE database of citations and abstracts of biomedical research articles) and Web of Science (online scientific information service, provided by Thomson Reuters, integrated in ISI Web of Knowledge, WOK, containing original articles based on clinical trials) databases were used to obtain all the data used in this study. The keywords used were: ("cancer patients" OR "cancer survivors") AND ("fatigue" OR "cancer-related fatigue") AND "exercise" AND "randomized controlled trial" AND "free full text" AND "custom date range" ("2001 January 1st to 2019 September 1st"). The search string was ("cancer patients" [All Fields] OR "cancer survivors" [All Fields]) AND ("fatigue" [All Fields] OR "cancerrelated fatigue" [All Fields]) AND "exercise". There was a custom date range published from 2001 January $1^{\text {st }}$ to 2019 September 1 st.

\section{Eligibility criteria}

Table 1. PICOST eligibility criteria.

\begin{tabular}{|c|c|}
\hline Parameter & $\begin{array}{cc}\text { Inclusion criteria } & \text { Exclusion criteria } \\
\end{array}$ \\
\hline Patients & $\begin{array}{l}\text { Adults ( } \geq 18 \text { years) living with or Patients }<18 \text { years } \\
\text { beyond cancer diagnosis not Patients receiving palliative care } \\
\text { receiving palliative care, Patients without cancer } \\
\text { undergoing or post therapy and/or Patients with unrecoverable } \\
\text { treatment metastatic disease }\end{array}$ \\
\hline Intervention & $\begin{array}{ll} & \text { Yoga, Dance, Qigong, Powerlifting, } \\
& \text { Tai chi, Pilates, Mindfulness, } \\
\text { Physical activity with AE, RE, EN Chess, Physiotherapy, Music } \\
\text { and/or combined exercises } & \text { therapy, CBT, EMS, Isometric } \\
& \text { training, Psychoeducation, Diet and } \\
& \text { Dietary supplements }\end{array}$ \\
\hline Comparator & $\begin{array}{l}\begin{array}{l}\text { Non-exercise control group; } \mathrm{RE}, \mathrm{AE} \\
\text { or EN exercise group }\end{array} \\
\begin{array}{l}\text { Non-exercise control group } \\
\text { receiving additional care, such as } \\
\text { diet, CBT, physiotherapy, etc. }\end{array}\end{array}$ \\
\hline Outcome & $\begin{array}{l}\text { Perceived of Fatigue measured by No fatigue result } \\
\text { self-report questionnaire }\end{array}$ \\
\hline Study design & $\begin{array}{ll}\text { RCTs with length of follow up of } 5 \text { Non-RCTs } \\
\text { RCTs lasted } 4 \text { weeks or less } \\
\text { weeks or more } & \text { No English language }\end{array}$ \\
\hline Timing & $\begin{array}{l}\text { From } 2001 \text { January } 1^{\text {st }} \text { to } 2019 \text { Before } 2001 \text { and after } 2019 \\
\text { September } 1^{\text {st }} \\
\text { September } 1^{\text {st }}\end{array}$ \\
\hline
\end{tabular}

Abbreviations: AE, Aerobic; RE, Resistance; EN, Endurance; CBT, Cognitive Behavioural Therapy; EMS, Electric Muscular Stimulation; RCTs, Randomized Controlled Trials. 
A summary of the inclusion and exclusion criteria for this review is shown in PICOST (Miller, 2001) Table 1. Each of the identified articles was independently analysed by three researchers. Patients were adults $(\geq 18$ years) living with, or beyond, any cancer diagnosis, undergoing or after some therapy and/or treatment; they didn't receive palliative care but active treatment as surgery, radiotherapy (included ADT), chemotherapy and/or hormone therapy. Metastatic diseases were included if it was not unrecoverable. We included RCTs in which were Aerobic, Resistance, Endurance and/or combined exercise. We examined only RCT in which were CRF outcomes. Only Randomized Controlled Trials were analysed, which were published from 2001 January $1^{\text {st }}$ to 2019 September $1^{\text {st }}$. We considered acceptable RCT which lasted 5 weeks or more and had one or multiple follow-ups for inclusion. We considerable acceptable all intensity of exercise if it was correctly reported. We excluded Yoga, Qigong, Tai chi, Powerlifting, Pilates and Dance exercises because they need a specialist trainer and some of them request a good motor activity experience. Every study had at least one control group, who didn't exercise, and could compare two or more group if it was a 3-armed trial. Outcomes had to underline changes in fatigue and were expressed in quantitative measures by a validated self-report questionnaire. Only English language was acceptable for inclusion study.

\section{Data collection process and analysis}

Two independent reviewers extracted data available in the full free texts. All variables for which data were sought are: a) Fatigue severity, measured by self-report questionnaire validated to assess and manage CRF in patients or survivors and expressed in $p$-value; b) Number of patients of interventions or control groups, sample mean age, gender of patients; c) Type of cancer and treatment; d) Type of exercise, its intensity and its frequency; e) Length of study analysis; f) Intervention in groups.

The principal summary measures were expressed as standardizes difference in means (Cohen's $d$ ) of CRF and $p$-value to quantify the idea of statistical significance of evidence. It is assumed that an effect size $d \geq$ 0.80 represents a large effect, $0.50 \leq d<0.80$ medium and $d<0.50$ small (Cohen, 1988). $P$-value is generally considered an $a=.05$ as a threshold value under which data has a statistical significance (Wasserstein \& Lazar., 2016).

\section{Additional analyses}

We analysed 4 different interventions:

1. Aerobic exercises as walking, jogging, swimming, biking and running. For stay in aerobic training, level of intensity was between $40-65 \% \mathrm{HR}_{\max }, 50-75 \% \mathrm{HR}_{\mathrm{r}}$, or $35-50 \% \mathrm{VO}_{2 \text { peak. }}$.

2. Resistance exercises as muscular contraction on machine or with resistance band. The intensity wasn't over 85\% 1RM.

3. Endurance exercises that trained cardiovascular and respiratory system with high intensity (> $80 \%$ $\mathrm{HR}_{\mathrm{r}}$ or $75 \%<\mathrm{VO}_{2 \text { peak }}<95 \%$ )

4. Combined two or more of precedent interventions.

\section{RESULTS}

\section{Study selection}

The search strategy found 1880 RCTs, of which 827 were free full texts. After checking for duplicates, remained 651 RCTs. From these, 47 trials were included for independent screening. 32 trials were excluded and only 15 respected the inclusion criteria (reported in Table 1) and were included in the systematic review. The PRISMA flow diagram (Moher et al., 2009) reported in Figure 1 reports an overview of "study selection". 

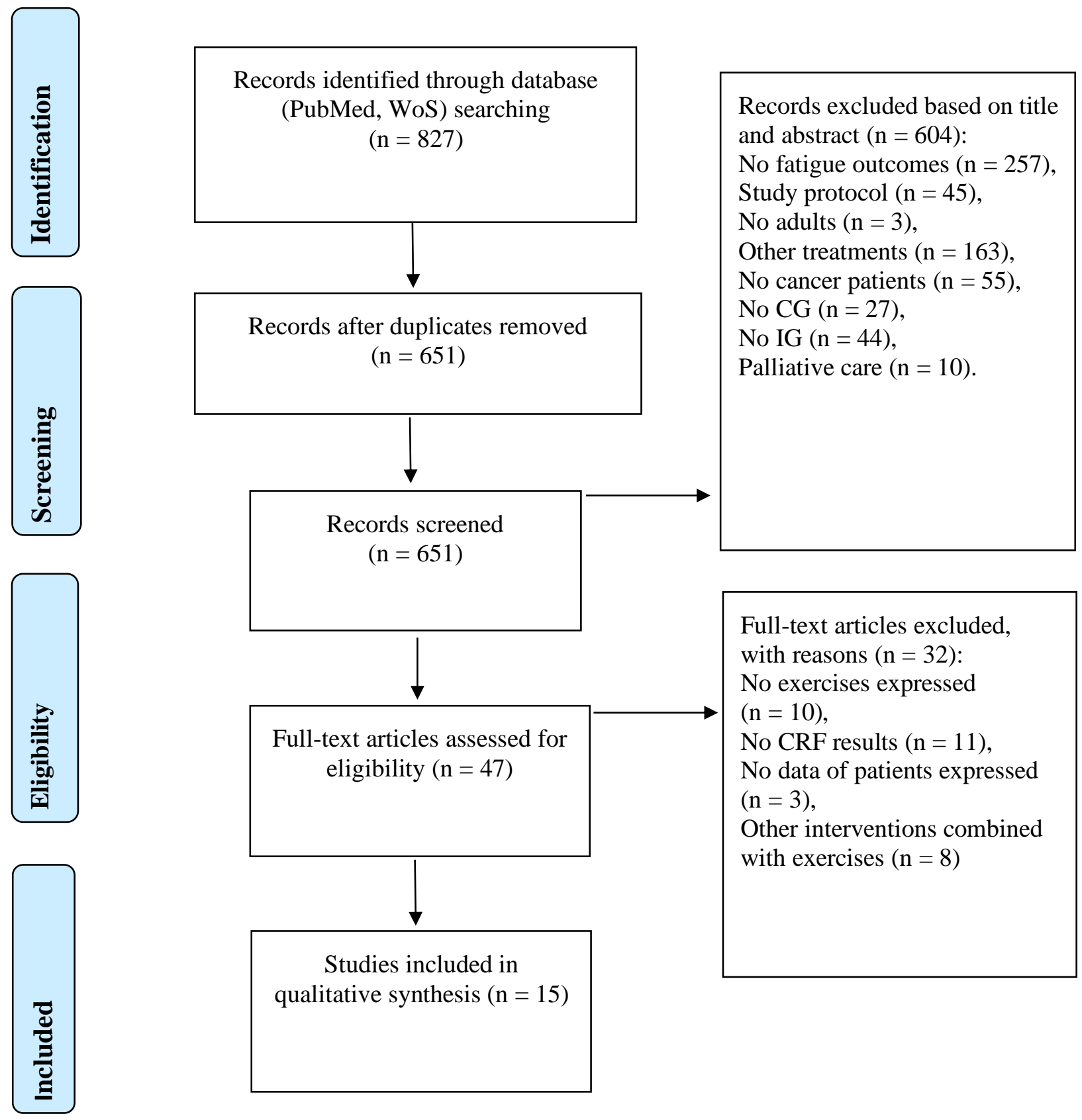

Figure 1. Flowchart on the literature search of exercise effects on cancer-related fatigue.

\section{Study characteristics}

All 15 trials were RCTs (Adams et al., 2018; Cormie et al., 2015; Dieli-Conwright et al., 2018; Galiano-Castillo et al., 2016; Hojan et al., 2016; Hwang et al., 2008; Kampshoff et al., 2015; Monga et al., 2007; Paulo et al., 2019; Penttinen et al., 2019; Pinto et al., 2005; Schmidt et al., 2015; Segal et al., 2009; Shobeiri et al., 2016; Steindorf et al., 2014). Table 2 reviews all patient details. In total, 1664 patients were included in the trials, of whom 376 were men and 1288 were women. 905 patients were randomized in an intervention group (IG1+IG2) and 749 were randomized in a control group of which 36 received a basic recommendation for exercise (Galiano-Castillo et al., 2016) and 142 followed relaxation treatment (Paulo et al., 2019; Schmidt et al., 2015; Steindorf et al., 2014). The weighted average age was 54.91. In 9 RCTs patients reported breast 
cancer (Dieli-Conwright et al., 2018; Galiano-Castillo et al., 2016; Hwang et al., 2008; Paulo et al., 2019; Penttinen et al., 2019; Pinto et al., 2005; Schmidt et al., 2015; Shobeiri et al., 2016; Steindorf et al., 2014), in 4 reported prostate cancer (Cormie et al., 2015; Hojan et al., 2016; Monga et al., 2007; Segal et al., 2009), in 1 reported testicular cancer (Adams et al., 2018), in 1 multiple cancer (Kampshoff et al., 2015). In 8 trials patients were still undergoing different therapies (Cormie et al., 2015; Hojan et al., 2016; Hwang et al., 2008; Monga et al., 2007; Paulo et al., 2019; Schmidt et al., 2015; Segal et al., 2009; Steindorf et al., 2014), in 3 trials patients completed therapies (Kampshoff et al., 2015; Penttinen et al., 2019; Shobeiri et al., 2016) and in 4 trials patients were analysed after therapies (Adams et al., 2018; Dieli-Conwright et al., 2018; GalianoCastillo et al., 2016; Pinto et al., 2005). Exercise interventions lasted from 5 weeks (Mendes et al., 2018) to 5 years (Penttinen et al., 2019), of which 11 trials did follow-up at baseline and after treatment (Adams et al., 2018; Cormie et al., 2015; Dieli-Conwright et al., 2018; Galiano-Castillo et al., 2016; Hojan et al., 2016; Hwang et al., 2008; Kampshoff et al., 2015; Pinto et al., 2005; Shobeiri et al., 2016; Schmidt et al., 2015), 3 RCTs did 3 different follow-up (baseline, T1 and T2) (Paulo et al., 2019; Segal et al., 2009; Steindorf et al., 2014) and other one trial did 6 follow-ups (Penttinen et al., 2019).

Table 2. Patients details.

\begin{tabular}{lcccccc}
\hline Study & Patients & IG1 & IG2 & CG & Age (yrs.) & Gender \\
\hline Paulo et al. (2019) & 36 & 18 & 0 & 18 & 65 & female \\
Shobeiri et al. (2016) & 53 & 27 & 0 & 26 & 43 & female \\
Galiano-Castillo et al. (2016) & 72 & 36 & 0 & 36 & 48 & female \\
Schmidt et al. (2015) & 95 & 49 & 0 & 46 & 53 & female \\
Adams et al. (2018) & 63 & 35 & 0 & 28 & 44 & male \\
Penttinen et al. (2019) & 444 & 235 & 0 & 209 & 53 & female \\
Dieli-Conwright et al. (2018) & 91 & 46 & 0 & 45 & 53 & female \\
Steindorf et al. (2014) & 155 & 77 & 0 & 78 & 56 & female \\
Kampshoff et al. (2015) & 277 & 91 & 95 & 91 & 54 & $\mathrm{f}=223 ; \mathrm{m}=54$ \\
Hojan et al. (2016) & 54 & 27 & 0 & 27 & 68.5 & male \\
Monga et al. (2007) & 21 & 11 & 0 & 10 & 69 & male \\
Segal et al. (2009) & 121 & 40 & 40 & 41 & 66 & male \\
Hwang et al. (2008) & 37 & 17 & 0 & 20 & 46 & female \\
Cormie et al. (2015) & 63 & 32 & 0 & 31 & 68.5 & male \\
Pinto et al. (2005) & 82 & 39 & 0 & 43 & 53 & female \\
ALL & 1664 & 780 & 135 & 749 & $54.91^{*}$ & $\mathrm{f}=1288 ; \mathrm{m}=376$ \\
\hline
\end{tabular}

*Weighted average of age; IG, intervention group; CG, control group; $m$, male; $f$, female.

\section{Results of individuals studies}

The results are summarized in Table 3. Thirteen RCTs found improvements in CRF (Adams et al., 2018; Cormie et al., 2015; Dieli-Conwright et al., 2018; Galiano-Castillo et al., 2016; Hojan et al., 2016; Hwang et al., 2008; Kampshoff et al., 2015; Monga et al., 2007; Paulo et al., 2019; Pinto et al., 2005; Segal et al., 2009; Shobeiri et al., 2016; Steindorf et al., 2014), one trial found improvements only in patients who were not affected of depression (Schmidt et al., 2015) and other one trial found improvements in patients who changed intensity of exercise or physical performance (Penttinen et al., 2019). Four types of exercise intervention were used: Aerobic (Monga et al., 2007; Pinto et al., 2005; Segal et al., 2009; Shobeiri et al., 2016); Resistance (Schmidt et al., 2015; Segal et al., 2009; Steindorf et al., 2014); Endurance (i.e., High Intensity Interval Training) (Adams et al., 2018), Combined (Cormie et al., 2015; Dieli-Conwright et al., 2018; GalianoCastillo et al., 2016; Hojan et al., 2016; Kampshoff et al., 2015; Mendes et al., 2018; Paulo et al., 2019; Penttinen et al., 2019). One of these trials (Segal et al., 2009) found improvements in CRF with aerobic 
Table 3. Studies that investigated the effects of exercise on cancer-related fatigue.

\begin{tabular}{|c|c|c|c|c|c|c|c|}
\hline Article & $\begin{array}{l}-n \text { IG1 } \\
-n \text { IG2 } \\
-n \text { CG } \\
- \text { Age (sm) } \\
- \text { Sex }\end{array}$ & $\begin{array}{l}\text {-Cancer } \\
\text {-Treatment }\end{array}$ & $\begin{array}{l}\text {-Outcome } \\
\text { instrument } \\
\text {-P.Value }\end{array}$ & $\begin{array}{l}\text {-Exercise } \\
\text {-Intensity } \\
\text {-Frequency }\end{array}$ & $\begin{array}{l}\text {-Time follow-up } \\
\text {-All Outcomes compared }\end{array}$ & Activity IG & Control Group \\
\hline $\begin{array}{l}\text { RCT }(2 \times 3) \text { Paulo } \\
\text { et al. (2019) }\end{array}$ & $\begin{array}{l}-n=18 \\
-n=\varnothing \\
-n=18 \\
-65 \text { years } \\
\text {-female }\end{array}$ & $\begin{array}{l}\text {-Breast }(100 \%) \\
\text {-Undergoing } \\
\text { aromatase } \\
\text { inhibition } \\
\text { therapy }\end{array}$ & $\begin{array}{l}-E O R T C Q L Q-30 \\
-p<.001 \text { (IG vs. } \\
\text { CG) }\end{array}$ & $\begin{array}{l}-A E+R E \\
-60-85 \% H_{\max }(A E) \\
-M a x \operatorname{rep}(R E) \\
-3 d / w\end{array}$ & $\begin{array}{l}\text {-9 Months } \\
\text {-Improved: } \\
\text { CRF, Nausea, Dyspnoea, } \\
\text { Sleep disturbance, } \\
\text { Physical fitness, etc. }\end{array}$ & $\begin{array}{l}\text { Warm-up + 40' RE exercises on } \\
\text { machine }+30^{\prime} \text { AE exercise + cool- } \\
\text { down }\end{array}$ & $\begin{array}{l}45^{\prime} \text { of Stretch and } \\
\text { relaxation exercise, } 2 \\
d / w\end{array}$ \\
\hline $\begin{array}{l}\text { RCT (2x2) } \\
\text { Shobeiri et al. } \\
(2016)\end{array}$ & $\begin{array}{l}-n=27 \\
-n=\varnothing \\
-n=26 \\
-43 \text { years } \\
\text {-female }\end{array}$ & $\begin{array}{l}\text {-Breast }(100 \%) \\
\text {-Completed } \\
\text { surgery and RT } \\
\text { o CT }\end{array}$ & $\begin{array}{l}-E O R T C Q L Q-30 \\
-p<.001 \text { (IG vs. } \\
C G)\end{array}$ & $\begin{array}{l}-A E \\
-50-75 \% H R_{r} \\
-2 d / w\end{array}$ & $\begin{array}{l}-10 \text { Weeks } \\
\text {-Improved: CRF, Nausea, } \\
\text { Pain, Dyspnoea, Insomnia, } \\
\text { physical and mental fitness } \\
\text { and QoL }\end{array}$ & $\begin{array}{l}\text { Warm-up (5-10' of stretching and } \\
\text { moderate walking })+15 \text { of } A E \\
\text { exercise }+5 \text { ' of cool down }\end{array}$ & $\begin{array}{l}\text { Usual Care and } \\
\text { sedentary lifestyle }\end{array}$ \\
\hline $\begin{array}{l}\text { RCT (2X2) } \\
\text { Galiano-Castillo } \\
\text { et al. (2016) }\end{array}$ & $\begin{array}{l}-n=36 \\
-n=\varnothing \\
-n=36 \\
-48 \text { years } \\
\text {-female }\end{array}$ & $\begin{array}{l}\text {-Breast }(100 \%) \\
\text {-After RT } \\
\text { and/or CT }\end{array}$ & $\begin{array}{l}-P F S \\
-p<.05 \text { (IG vs CG) }\end{array}$ & $\begin{array}{l}-A E+R E \\
-A C S M \text { guidelines } \\
-3 d / w\end{array}$ & $\begin{array}{l}\text {-6 Months } \\
\text {-Improved: CRF, Health } \\
\text { status, Physical and } \\
\text { Cognitive fitness }\end{array}$ & $\begin{array}{l}90 \text { ' of training with warm-up }+\mathrm{AE} \\
\text { and RE exercises + cool down }\end{array}$ & $\begin{array}{l}\text { Basic recommendation } \\
\text { for exercises }\end{array}$ \\
\hline $\begin{array}{l}\text { RCT (2x2) } \\
\text { Schmidt et al. } \\
(2015)\end{array}$ & $\begin{array}{l}-n=49 \\
-n=\varnothing \\
-n=46 \\
-53 \text { years } \\
\text {-female }\end{array}$ & $\begin{array}{l}\text {-Breast }(100 \%) \\
\text {-Undergoing } \\
\text { adjuvant CT }\end{array}$ & $\begin{array}{l}-\mathrm{FAQ} \\
-p<.098 \text { (patients } \\
\text { with high Iv of } \\
\text { depression) } \\
-p<.038 \text { (patients } \\
\text { without depression) }\end{array}$ & $\begin{array}{l}\text {-RE } \\
-60-80 \% 1 R M \\
-2 d / w\end{array}$ & $\begin{array}{l}-12 \text { Weeks } \\
\text {-Improved: CRF, social and } \\
\text { role f. }\end{array}$ & $\begin{array}{l}8 \text { machines based progressive RE } \\
\text { training ( } 3 \text { sets } x 12 \text { repetition); no } \\
\text { AE exercises }\end{array}$ & $\begin{array}{l}\text { Relaxation accord to } \\
\text { Jacobsen (Jacobsen \& } \\
\text { al., 1999) }\end{array}$ \\
\hline $\begin{array}{l}\text { RCT (2X2) } \\
\text { Adams et al. } \\
(2018)\end{array}$ & $\begin{array}{l}-n=35 \\
-n=\varnothing \\
-n=28 \\
-44 \text { years } \\
- \text { male }\end{array}$ & $\begin{array}{l}\text {-Testicular } \\
(100 \%) \\
\text {-After RT, CT } \\
\text { or others }\end{array}$ & $\begin{array}{l}-F A C T-F \\
-p=.003\end{array}$ & $\begin{array}{l}\text {-High Intensity } \\
\text { Interval-Training } \\
-75-95 \% \mathrm{VO}_{2 \max } \\
-3 \mathrm{~d} / \mathrm{w}\end{array}$ & $\begin{array}{l}\text {-3 Months } \\
\text {-Improved: CRF, Mental } \\
\text { component and vitality }\end{array}$ & $\begin{array}{l}\text { Warm-up 5' + } \\
\text { HIIT } 25^{\prime}(4 \text { sets x 4', 3' of active } \\
\text { recovery in } 3 \text { intervals) + cool } \\
\text { down }\end{array}$ & $\begin{array}{l}\text { Maintained baseline } \\
\text { exercise Iv }\end{array}$ \\
\hline $\begin{array}{l}\text { RCT * } \\
\text { Penttinen et al. } \\
(2019)\end{array}$ & $\begin{array}{l}-n=235 \\
-n=\varnothing \\
-n=209 \\
-53 \text { years } \\
\text {-female }\end{array}$ & $\begin{array}{l}\text {-Breast }(100 \%) \\
\text {-Completed CT } \\
\text { and RT or } \\
\text { started ET }\end{array}$ & $\begin{array}{l}-\mathrm{FACIT-F} \\
-p=.065 \text { (in patients } \\
\text { without increment of } \\
\text { MET-h/w) }\end{array}$ & $\begin{array}{l}-\mathrm{AE}+\mathrm{RE}+\mathrm{EN} \\
-\mathrm{RPE} 14-16 \\
\text {-Incremented MET- } \\
\mathrm{h} / \mathrm{w} \text { or changed } \\
\text { physical } \\
\text { performance }\end{array}$ & $\begin{array}{l}-5 \text { Years } \\
\text {-Improved: CRF, Physical } \\
\text { and Role fitness }\end{array}$ & $\begin{array}{l}1 \text { day with } 60 \text { ' of } A E \text { exercises and } \\
\text { circuit training } \\
2 \text { days (at least) of EN exercise }\end{array}$ & $\begin{array}{l}\text { Maintained baseline } \\
\text { exercises Iv }\end{array}$ \\
\hline
\end{tabular}




\begin{tabular}{|c|c|c|c|c|c|c|c|}
\hline & & & $\begin{array}{l}-p=.002 \text { (in patients } \\
\text { with increment of } \\
\text { MET-h/w) } \\
-p=.003 \text { (patients } \\
\text { who changed } p . \\
\text { performance) }\end{array}$ & 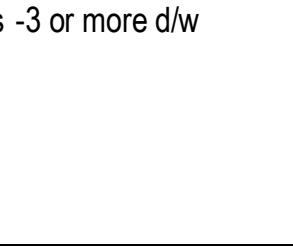 & & & \\
\hline $\begin{array}{l}\text { RCT (2x2) Dieli- } \\
\text { Conwright et al. } \\
\text { (2018) }\end{array}$ & $\begin{array}{l}-n=46 \\
-n=\varnothing \\
-n=45 \\
-63 \text { years } \\
\text {-female }\end{array}$ & $\begin{array}{l}\text {-Breast }(100 \%) \\
\text {-After CT e RT }\end{array}$ & $\begin{array}{l}-\mathrm{BFI} \\
-p<.001 \text { (IG vs CG) }\end{array}$ & $\begin{array}{l}-A E+R E \\
-65-80 \% H_{\max } \\
-60-80 \% 1 R M \\
\text { (weight increased by } \\
10-15 \% \text { ) } \\
-3 \mathrm{~d} / \mathrm{w}\end{array}$ & $\begin{array}{l}\text {-12 Weeks } \\
\text {-Improved: CRF, Physical } \\
\text { fitness, QoL }\end{array}$ & $\begin{array}{l}\text { Day } 1 \text { and } 3: 5^{\prime} \text { warm-up }+70^{\prime} \text { RE } \\
\text { and } A E \text { exercises }+5^{\prime} \text { cool down } \\
\text { Day } 2: 30-50^{\prime} \text { of } A E \text { exercises }\end{array}$ & Usual care \\
\hline $\begin{array}{l}\mathrm{RCT}(2 \times 3) \\
\text { Steindorf et al. } \\
(2014)\end{array}$ & $\begin{array}{l}-n=77 \\
-n=\varnothing \\
-n=78 \\
-56 \text { years } \\
\text {-female }\end{array}$ & $\begin{array}{l}\text {-Breast }(100 \%) \\
\text {-Undergoing } \\
\text { adjuvant RT }\end{array}$ & $\begin{array}{l}-F A Q \\
-p=.044\end{array}$ & $\begin{array}{l}-\mathrm{RE} \\
-60-80 \% 1 \mathrm{RM} \\
-2 \mathrm{~d} / \mathrm{w}\end{array}$ & $\begin{array}{l}\text {-12 Weeks } \\
\text { - Improved: CRF, Pain }\end{array}$ & $\begin{array}{l}60 \text { ' with } 8 \text { different machine-based } \\
\text { RE exercise ( } 3 \text { sets of } 8-12 \text { reps) }\end{array}$ & $\begin{array}{l}\text { Relaxation without RE } \\
\text { and } A E \text { exercises }\end{array}$ \\
\hline $\begin{array}{l}\text { RCT }(2 \times 2) \text { Hojan } \\
\text { et al. }(2016)\end{array}$ & $\begin{array}{l}-n=27 \\
-n=\varnothing \\
-n=27 \\
-68.5 \text { years } \\
- \text { male }\end{array}$ & $\begin{array}{l}\text {-Prostate } \\
(100 \%) \\
\text {-Undergoing } \\
\text { RT }\end{array}$ & $\begin{array}{l}-F A C T-F \\
-p<.001\end{array}$ & $\begin{array}{l}-A E+R E \\
-65-70 \% H_{r} \\
-70-75 \% 1 R M \\
-5 d / w\end{array}$ & $\begin{array}{l}\text {-3-5 Months } \\
\text {-Improved: CRF, Physical } \\
\text { fitness and Well-being }\end{array}$ & $\begin{array}{l}\text { 5' warm-up + 30' AE exercise + } 15 \\
\text { RE exercise }+5 \text { ' cool down }\end{array}$ & $\begin{array}{l}\text { Activity of daily living } \\
\text { without physical } \\
\text { exercises }\end{array}$ \\
\hline $\begin{array}{l}\text { RCT }(3 \times 2) \\
\text { Kampshoff et } \\
\text { al. }(2015)\end{array}$ & $\begin{array}{l}-n=91 \\
(\mathrm{HI}) \\
-n=95 \\
(\mathrm{LMI}) \\
-n= \\
91(\mathrm{WLC}) \\
-54 \text { years } \\
- \text { male } \\
(20 \%) \\
- \text { female } \\
(80 \%) \\
\end{array}$ & $\begin{array}{l}\text {-Breast }(65 \%) \text {, } \\
\text {-Colon } \\
(17.7 \%), \\
\text {-Ovarian } \\
(4.3 \%), \\
\text {-Lymphoma } \\
(9.4 \%), \\
\text {-Cervix }(1.7 \%) \\
\text {-Testis }(1.9 \%) \\
\text {-Completed CT }\end{array}$ & $\begin{array}{l}-M F I \\
-p<.05 \text { (HI vs WLC) } \\
-p<.05 \text { (LMI vs } \\
\text { WLC) }\end{array}$ & $\begin{array}{l}-\mathrm{RE}+\mathrm{EN} \\
-\mathrm{HI} 70-85 \% 1 \mathrm{RM} \\
\text { and } \geq 80 \% \mathrm{HR}_{\mathrm{r}} \\
-\mathrm{LMI} 40-55 \% 1 \mathrm{RM} \\
\text { and } 40-55 \% \mathrm{HRr}\end{array}$ & $\begin{array}{l}\text { - } 12 \text { Weeks } \\
\text {-Improved CRF (HI and } \\
\text { LMI) and Physical f. (HI } \\
\text { and LMI) }\end{array}$ & $\begin{array}{l}6 \text { RE exercises for LMI and HI (2 } \\
\text { sets of } 10 \text { rep) } \\
2 \times 8^{\prime} \text { at different workload for LMI } \\
\text { and HI }\end{array}$ & Wait list control \\
\hline $\begin{array}{l}\text { RCT }(2 \times 2) \\
\text { Monga et al. } \\
(2007)\end{array}$ & $\begin{array}{l}-n=11 \\
-n=\varnothing \\
-n=10 \\
-69 \text { years } \\
- \text { male }\end{array}$ & $\begin{array}{l}\text {-Prostate } \\
(100 \%) \\
\text {-Undergoing } \\
\text { RT }\end{array}$ & $\begin{array}{l}-P F S \\
-p<.001\end{array}$ & $\begin{array}{l}-A E \\
- \text { Maintained target } \\
\text { HR } \\
-2 \text { or more } d / w\end{array}$ & $\begin{array}{l}-8 \text { Weeks } \\
\text {-Improved: CRF, Physical } \\
\text { performance and Well- } \\
\text { being }\end{array}$ & $\begin{array}{l}\text { 10' warm-up + } 30 \text { ' AE (walking) + } \\
5-10^{\prime} \text { cool down }\end{array}$ & $\begin{array}{l}\text { Usual care without } \\
\text { exercises }\end{array}$ \\
\hline
\end{tabular}




\begin{tabular}{|c|c|c|c|c|c|c|c|}
\hline $\begin{array}{l}\text { RCT }(3 \times 3) \text { Segal } \\
\text { et al. }(2009)\end{array}$ & $\begin{array}{l}-n=40 \\
(R E) \\
-n=40 \\
(A E) \\
-n=41 \\
(C G) \\
-66 \text { years } \\
\text {-male }\end{array}$ & $\begin{array}{l}\text {-Prostate } \\
(100 \%) \\
\text {-Undergoing } \\
\text { RT }\end{array}$ & $\begin{array}{l}-F A C T-F \\
-p<.004 \text { (AE vs CG } \\
\text { at first follow-up) } \\
-p<.002 \text { (RE vs CG } \\
\text { at last follow-up) }\end{array}$ & $\begin{array}{l}-\mathrm{RE} \text { or } \mathrm{AE} \\
-60-70 \% 1 \mathrm{RM} \\
-50-75 \% \mathrm{VO}_{2 \max } \\
-3 \mathrm{~d} / \mathrm{w}\end{array}$ & $\begin{array}{l}-12-24 \text { Weeks } \\
\text {-Improved: CRF (AE vs CG } \\
\text { at week 12; RE vs CG at } \\
\text { week } 24 \text { ), etc. }\end{array}$ & $\begin{array}{l}5 \text { ' warm-up + } 10 \text { different } \\
\text { exercises ( } 2 \text { sets of } 8-12 \text { rep) }+15 \text { - } \\
45^{\prime} \text { of } A E \text { exercises (cycle } \\
\text { ergometer, treadmill or elliptical + } \\
5 \text { ' cool down }\end{array}$ & Usual care \\
\hline $\begin{array}{l}\text { RCT }(2 \times 2) \\
\text { Hwang et al. } \\
(2008)\end{array}$ & $\begin{array}{l}-n=17 \\
-n=\varnothing \\
-n=20 \\
-46 \text { years } \\
\text {-female }\end{array}$ & $\begin{array}{l}\text {-Breast }(100 \%) \\
\text {-Undergoing } \\
\text { RT }\end{array}$ & $\begin{array}{l}-\mathrm{BFI} \\
-\mathrm{p}<.05\end{array}$ & $\begin{array}{l}-A E+R E \\
-50-70 \% H R_{\max } \\
-3 d / w\end{array}$ & \multicolumn{3}{|c|}{$\begin{array}{l}\text {-Improved CRF, QoL, Pain, exercises } \\
\text { ROM }\end{array}$} \\
\hline $\begin{array}{l}\mathrm{RCT}(2 \times 2) \\
\text { Cormie et al. } \\
(2014)\end{array}$ & $\begin{array}{l}-n=32 \\
-n=\varnothing \\
-n=31 \\
-68.5 \text { years } \\
- \text { male }\end{array}$ & $\begin{array}{l}\text {-Prostate } \\
(100 \%) \\
\text {-Undergoing } \\
\text { ADT }\end{array}$ & $\begin{array}{l}-\mathrm{FACIT-F} \\
-p=.042\end{array}$ & $\begin{array}{l}-A E+R E \\
-70-85 \% H R_{\max } \\
-60-85 \% 1 R M \\
-2 d / w\end{array}$ & $\begin{array}{l}-3 \text { Months } \\
\text {-Improved CRF, Mental } \\
\text { health, Sexual and } \\
\text { Physical fitness, Body } \\
\text { composition }\end{array}$ & $\begin{array}{l}\text { Warm-up + 20-30' of AE exercises } \\
+8 \text { RE exercises (1-4 sets of } 6-12 \\
\text { rep) + cool down. Total of } 60^{\prime}\end{array}$ & Usual care \\
\hline $\begin{array}{l}\text { RCT }(2 \times 2) \text { Pinto } \\
\text { et al. }(2005)\end{array}$ & $\begin{array}{l}-n=39 \\
-n=\varnothing \\
-n=43 \\
-53 \text { years } \\
\text {-female }\end{array}$ & $\begin{array}{l}\text {-Breast }(100 \%) \\
\text {-After RT, CT } \\
\text { and/or HT }\end{array}$ & $\begin{array}{l}-\mathrm{LASA} \\
-p=.001\end{array}$ & $\begin{array}{l}-A E \\
-55-65 \% H R_{\max } \\
-2 \text { to } 5 \mathrm{~d} / \mathrm{w}\end{array}$ & $\begin{array}{l}-12 \text { Weeks } \\
\text {-Improved: CRF and } \\
\text { Physical activity }\end{array}$ & 10-30' of AE exercises & $\begin{array}{l}\text { Maintained Iv of daily } \\
\text { activity }\end{array}$ \\
\hline $\begin{array}{l}{ }^{*} R C T \text { including } 3 \\
\text { Therapy; ADT, An } \\
\text { Functional Assess } \\
\text { Assessment } \text { Aues }\end{array}$ & sarogen De & $\begin{array}{l}\text { ronic Illness The } \\
\text { ASA, Linear An } \\
\text { rol; HIIT, High Ir }\end{array}$ & Abbreviations: $A E$, & Aerobic; RE, Resi & $\begin{array}{l}\text { e; CRF, Cancer Related Fa } \\
\text { for Research and Treatmen } \\
\text { ntory; PFS, Piper Fatigue So } \\
\text { roup; CG, Control Group; Sr }\end{array}$ & $\begin{array}{l}\text { atigue; RT, Radiotherapy; CT, Chem } \\
\text { nt of Cancer Quality of Life Question } \\
\text { Scale; MFI, Multidimensional Fatigue } \\
\text { Sm, Simple mean; HI, High Intensity; }\end{array}$ & $\begin{array}{l}\text { motherapy; HT, Hormc } \\
\text { nnaire-Core 30; FACI7 } \\
\text { Inventory; FAQ, Fatic } \\
\text { f: LMI, Low-To-Moder }\end{array}$ \\
\hline
\end{tabular}


exercise at first follow-up and with resistance exercise at last follow-up. Intensity of the exercise varied from low (Galiano-Castillo et al., 2016; Hwang et al., 2008; Pinto et al., 2005; Shobeiri et al., 2016) to moderate (Cormie et al., 2015; Dieli-Conwright et al., 2018; Hojan et al., 2016; Monga et al., 2007; Segal et al., 2009; Paulo et al., 2019; Schmidt et al., 2015; Steindorf et al., 2014) to high (Adams et al., 2018) or changed during treatment (Kampshoff et al., 2015; Penttinen et al., 2019). The most important outcomes were shown in two Aerobic treatment trials (Monga et al., 2007; Shobeiri et al., 2016) and two Combined treatment trials (DieliConwright et al., 2018; Paulo et al., 2019).

\section{Outcomes of RCTs}

The outcomes of trials are represented in Figure 2. Two RCTs didn't report Cohen's $d$ (Hwang et al., 2008; Penttinen et al., 2019). The two three-armed studies (Kampshoff et al., 2015; Penttinen \& al., 2019) showed different $p$-value for every experimental group confronted to control group. The most important effect size was found in four trials (Dieli-Conwright et al., 2018; Monga et al., 2007; Paulo et al., 2019; Shobeiri et al., 2016).

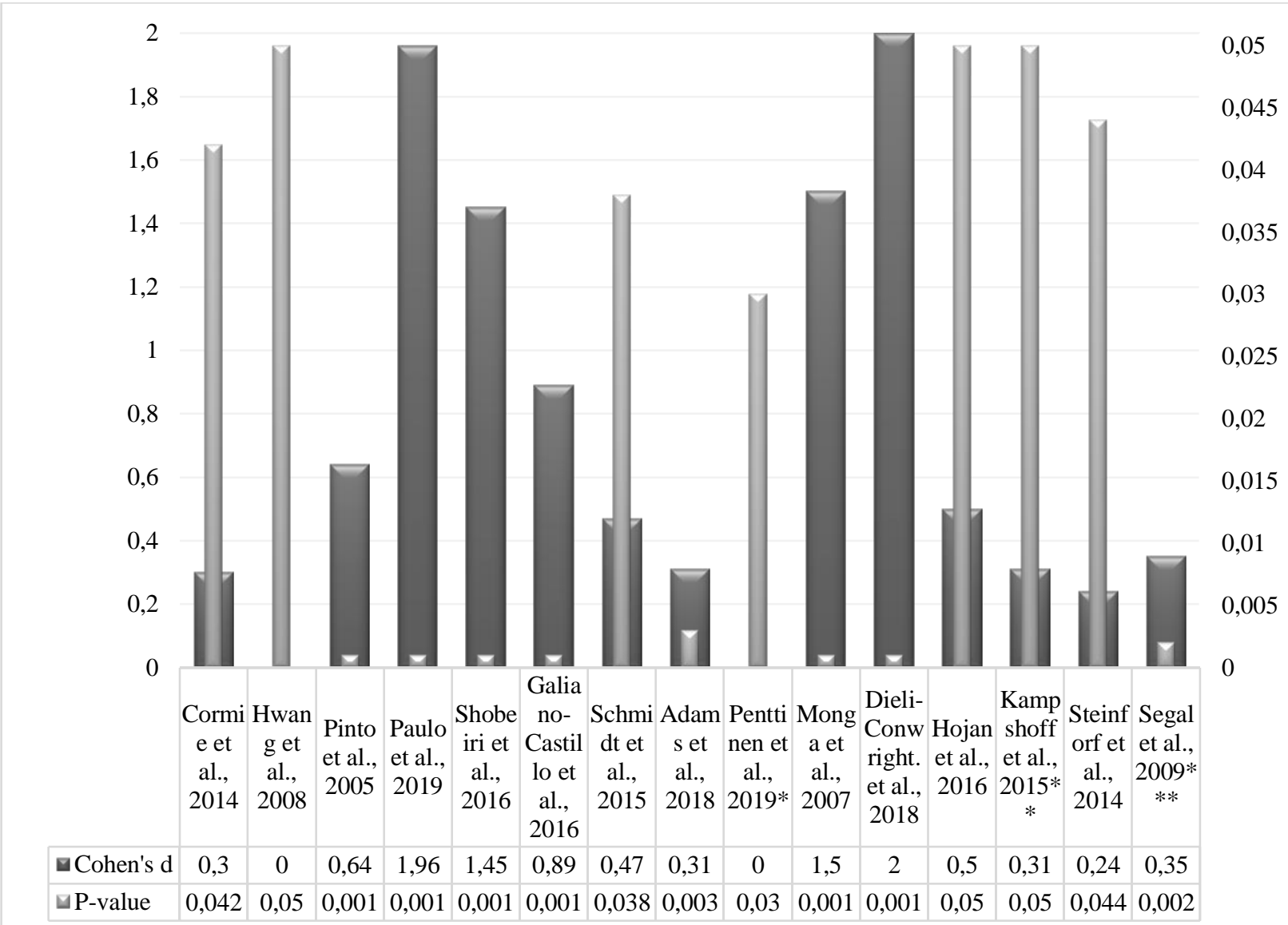

Where Cohen's $d$ results 0 we haven't found value in trial. *In this trial there were 2 different $p$-value: .03 for group who changed performance and .02 in group who incremented MET. ${ }^{* *}$ In this trial there were 2 different $p$-value and effect size: Low to Moderate Intensity reported $p<.05$ and $d=0.31$; High Intensity reported $p<.05$ and $d=0.35$. ${ }^{* * *}$ In this trial there where 2 different $p$-value and effect size: Resistance Training reported $p=.002$ and $d=0.35$; Aerobic Training reported $p=.080$ and $d=0.24$. Groups were analysed at 24 weeks follow-up versus Usual Care group.

Figure 2. Effect size and p-value of trials. 


\section{DISCUSSION}

\section{Summary of evidence}

This study aims to investigate the effect of exercise on CRF, how exercise was used during the last decade and which is the best exercise program in the cancer patient. The results reported in Table 3 and Figure 2 recommend that aerobic exercise improves CRF better than other treatments, but it also provides good outcomes combined with resistance training. To get the best results, the better way is doing exercises at least two days/week, for eight or more weeks. The studies in which are reported best improvements followed a low to moderate intensity of exercises, showing the same effects in men and women. Thus, according to NCCN (2015) and the American College of Sports Medicine (2018), physical activity makes better the quality of life in cancer patients and improves psychological and physiological fitness.

\section{Implication}

The positive effects found after an exercise treatment underline the importance of daily activities in the patient's life during cancer disease. The preventive intervention allows us to increase the wellbeing of cancer patients improving fatigue sensation and helping patients undergoing therapy to continue their activity of life. Furthermore, exercise programs can increase muscle strength, physical fitness, physical and psychological function, body composition and can positively impact biomarkers associated with cancer progression (Stout et al., 2017). Changes on CRF are associated with other factors as pain, insomnia, depression, anxiety, mood disturbance and physiological distress factors (Ahlberg et al., 2003). In a study of Fiuza-Luces et al. (2013), exercise training was considered as a "poly-pills" for its multiple effects (Stefani et al., 2016) and Van Waart et al. (2015) showed the beneficial outcomes observed in patients with cancer.

\section{Limitations}

The main limitations of this systematic review are:

- A small number of trials used to find exercise effects on CRF;

- A small number of cancer types. The most of patients had breast or prostate cancer;

- Some trials didn't report effect size;

- Researchers measured fatigue with different self-report scale and for different length; this can't afford us to advise a better way to select a design of research;

- Patients were undergoing different therapy (chemotherapy, radiotherapy, etc.) or they concluded it and we can't choose the best exercise treatment for every care;

- In some trial patients did exercises without the supervision of a specialist during the session; this is a limitation to quantify accurate data;

- Some trials reported several outcomes in follow-ups which were done at a different moment.

\section{CONCLUSIONS}

Based on this systematic review and the findings of the investigated studies shown in Table 3, we conclude that exercise improves CRF, especially with aerobic and combined programs. This systematic review can help the exercise professional to plan exercise sessions correctly and we recommend increasing quality and quantity of exercise in cancer patients by monitoring every parameter during sessions. Nevertheless, more studies are needed to value the effects of physical exercise training on cancer patients and CRF should be assessed as a primary outcome. 


\section{AUTHOR CONTRIBUTIONS}

SC, GG, MM and FF developed the research concept and study design. SC, GG, and MM performed the literature review and data collection. SC, GG and MM wrote the manuscript. GG and FF reviewed the manuscript. All authors contributed intellectually to the manuscript, and all authors have read the manuscript and approved the submission.

\section{SUPPORTING AGENCIES}

This research received no external funding.

\section{DISCLOSURE STATEMENT}

The authors declared no potential conflicts of interest concerning the research, authorship, and/or publication of this article.

\section{REFERENCES}

ACSM (2018). ACSM's Guidelines for Exercise Testing and Prescription (10th ed.). Baltimore: Lippincott Williams \& Wilkins.

Adams, S. C., DeLorey, D. S., Davenport, M. H., Fairey, A. S., North, S., \& Courneya, K. S. (2018). Effects of high-intensity interval training on fatigue and quality of life in testicular cancer survivors. British Journal of Cancer, 118(10), 1313-1321. https://doi.org/10.1038/s41416-018-0044-7

Ahlberg, K., Ekman, T., Gaston-Johansson, F., \& Mock, V. (2003). Assessment and management of cancer-related fatigue in adult. Lancet, 362, 640-650. https://doi.org/10.1016/s0140-6736(03)14186$\underline{4}$

AIOM , AIRTUM, PASSI. (2018). I numeri del cancro in Italia ( $9^{\wedge}$ ed.). Brescia: Intermedia Editore. https://www.aiom.it/wp-content/uploads/2018/10/2018 NumeriCancro-operatori.pdf

Akechi, T., Kugaya, A., Okamura, H., Yamawaki, S., \& Uchitomi, Y. (1999). Fatigue and its associated factors in ambulatory cancer patients: a prelilinary study. Journal of Pain and Symptom Management, 17(1), 42-48. https://doi.org/10.1016/s0885-3924(98)00105-5

American Cancer Society (2019). Cancer Facts \& Figure. Retrieved from https://www.cancer.org/content/dam/cancer-org/research/cancer-facts-and-statistics/annualcancer-facts-and-figures/2019/cancer-facts-and-figures-2019.pdf

Bernsten, S., Aaronson, N. K., Buffart, L., Börjeson, S., Demmelmaier, I., Hellbom, M., Hojman, P., Igelström, H., Johansson, B., Pingel, R., Raastad, T., Velikova, G., Åsenlöf, P., \& Nordin, K. (2017). Design of randomized controlled trial of physical training and cancer (Phys-Can)- the impact of exercise on cancer related fatigue, quality of life and disease outcome. BMC Cancer, 17(1), 218. https://doi.org/10.1186/s12885-017-3197-5

Borg, G. (1970). Perceived exertion and pain scales. Scandinavian Journal of Rehabilitation Medicine, 23, 92-96.

Bower, J., Ganz, P., \& Desmond, K. (2000). Fatigue in breast cancer survivors: occurrence, correlates, and impact on quality of life. Journal of Clinical Oncology, 18(4), 743-753. https://doi.org/10.1200/jco.2000.18.4.743

Broeckel, J., Jacobsen, P., Horton, Balducci, L., \& Lyman, G. H. (1998). Characteristics and correlates of fatigue after adjuvant chemotherapy for breast cancer. Journal of Clinical Oncology, 16(5), 16891696. https://doi.org/10.1200/ico.1998.16.5.1689 
Brown, D. J., McMillan, D. C., \& Milroy, R. (2005). The correlation between fatigue, physical function, the systemic inflammatory response, and psychological distress in patients with advanced lung cancer. Cancer, 103(2), 377-382. https://doi.org/10.1002/cncr.20777

Cataldi, S., Latino, F., Greco, G., \& Fischetti, F. (2019). Multilateral training improves physical fitness and fatigue perception in cancer patients. Journal of Human Sport and Exercise, 14(4proc), S910-S920. https://doi.org/10.14198/ihse.2019.14.proc4.54

Cella, D., Davis, K., Breitbart, W., \& Curt, G. (2001). Cancer-related fatigue: Prevalence of proposed diagnostic criteria in a United States sample of cancer survivors. Journal of Clinical Oncology, 19(14), 3385-3389. https://doi.org/10.1200/jco.2001.19.14.3385

Cheng, Y. J., Macera, C. A., Church, T. S., \& Blair, S. N. (2002). Heart rate reserve as a predictor of cardiovascular and all-cause mortality in men. Medicine \& Science in Sports \& Exercice, 34(12), 1873-1878. https://doi.org/10.1097/00005768-200212000-00003

Cink, R. E., \& Thomas, T. R. (1981, September). Validity of the Astrand-Ryhming nomogram for predicting maximal oxygen intake. British Journal of Sports Medicine, 15(3), 182-185. https://doi.org/10.1136/bjsm.15.3.182

Cleeland, C. S., Wang, X. S., \& Proceedings NCCN. (1999). Measuring and understanding fatigue. Oncology, 13, 91-97.

Cohen, J. (1988). Statistical Power Analysis for the Behavioral Sciences. New York: Routledge.

Cormie, P., Galvão, D. A., Spry, N., Joseph, D., Chee, R., \& Taaffe, D. R. (2015). Can supervised exercise prevent treatment toxicity in patients with prostate cancer initiating androgen-deprivation therapy: a randomised controlled trial. British Journal of Urology International, 115(2), pp. 256-266. https://doi.org/10.1111/bju.12646

Cramp, F., \& Byron-Daniel, J. (2012). Exercise for the management of cancer-related fatigue in adults. Cochrane Database Systematic Review, CD006145. https://doi.org/10.1002/14651858.cd006145.pub3

Curt, G. A., Breitbart, W., Cella, D., Groopman, J. E., Horning, S. J., Itri, L. M., Johnson, D. H., Miaskowski, C., Scherr, S. L., Portenoy, R. K., \& Vogelzang, N. J. (2000). Impact of cancer-related fatigue on the lives of patients: New findings from the Fatigue Coalition. Oncologist, 5(5), 353-360. https://doi.org/10.1634/theoncologist.5-5-353

Delorme, T. L., \& Watkins, A. L. (1948, May). Technics of progressive resistance exercise. Archives of Physical Medicine and Rehabilitation, 29(5), 263-273.

Dieli-Conwright, C. M., Courneya, K. S., Demark-Wahnefried, W., Sami, N., Lee, K., \& Sweeney F. C. (2018, October 19). Aerobic and resistance exercise improves physical fitness, bone health, and quality of life in overweight and obese breast cancer survivors: a randomized controlled trial. Breast Cancer Research, 20(1), 124. https://doi.org/10.1186/s13058-018-1051-6

Fischetti, F., Greco, G., Cataldi, S., Minoia, C., Loseto, G., \& Guarini, A. (2019). Effects of Physical Exercise Intervention on Psychological and Physical Fitness in Lymphoma Patients. Medicina, 55(7), 379. https://doi.org/10.3390/medicina55070379

Fiuza-Luces, C., Garatachea, N., Berger, N. A., \& Lucia, A. (2013, September). Exercise is the real polypill. Physiology (Bethesda), 28(5), 330-358. https://doi.org/10.1152/physiol.00019.2013

Forsyth, L. M., Preuss, H. G., MacDowell, A. L., Chiazze, L., Birkmayer, G. D., \& Bellanti, J. A. (1999). Therapeutic effects of oral NADH on the symptoms of patients with chronic fatigue syndrome. Annual of Allergy, Asthma \& Immunology, 82(2), 185-191. https://doi.org/10.1016/s1081-1206(10)62595-1

Galiano-Castillo, N., Cantarero-Villanueva, I., Fernàndez-Lao, C., Ariza-García, A., Díaz-Rodríguez L., \& Del-Moral-Ávila, R. (2016). Telehealth system: A randomized controlled trial evaluating the impact of an internet-based exercise intervention on quality of life, pain, muscle strength, and fatigue in breast cancer survivors. Cancer, 122(20), 3166-3174. https://doi.org/10.1002/cncr.30172 
Hofman, M., Ryan, J. L., Figueroa-Moseley, C. D., \& Jean-Pierre, P. (2007). Cancer-related fatigue: the scale of the problem. Oncologist, 12(Suppl 1), 4-10. https://doi.org/10.1634/theoncologist.12-s1-4

Hojan, K., Kwiatkowska-Borowczyk, E., Leporowska, E., Górecki, M., Ozga-Majchrzak, O., Milecki, T., \& Milecki, P. (2016). Physical exercise for functional capacity, blood immune function, fatigue, and quality of life in high-risk prostate cancer patients during radiotherapy: a prospective, randomized clinical study. European Journal of Physical and Rehabilitation Medicine, 52(4), 489-501. https://doi.org/10.1016/j.rehab.2014.03.1350

Humpel, N., \& Iverson, D. C. (2007). Depression and quality of life in cancer survivors: is there a relationship with physical activity? International Journal of Behavioral Nutrition and Physical Activity, 4, 65. https://doi.org/10.1186/1479-5868-4-65

Hwang, J. H., Chang, H. J., Shim, Y. H., Park, W. H., Park, W., Huh, S. J., \& Yang, J. H. (2008). Effects of supervised exercise therapy in patients receiving radiotherapy for breast cancer. Yonsei Medical Journal, 49(3), 443-450. https://doi.org/10.3349/ymj.2008.49.3.443

Isaksson, B., Rippe, C., Simonoska, R., Holm, J. E., Glaumann, H., Segersvärd, R., Larsson, J., Erlanson-Albertsson, C., \& Permert, J. (2002). Obstructive jaundice results in increased liver expression of uncoupling protein 2 and intact skeletal muscle glucose metabolism in the rat. $\begin{array}{llll}\text { Scandinavia Journal of } & \text { Gastroenterology, }\end{array}$ https://doi.org/10.1080/003655202753387446

Jacobsen, P. B., Hann, D. M., Azzarello, L. M., Horton, J., Balducci, L., \& Lyman, G. H. (1999). Fatigue in woman receiving adjuvant chemotherapy for breast cancer: characteristics, course and correlates. Journal of Pain and Symptom Management, 18(4), 233-242. https://doi.org/10.1016/s08853924(99)00082-2

Kampshoff, C. S., Chinapaw, M. J., Brug, J., Twisk, J. W., Schep, G., Nijziel, M. R., van Mechelen, W., \& Buffart, L. M. (2015). Randomized controlled trial of the effects of high intensity and low-tomoderate intensity exercise on physical fitness and fatigue in cancer survivors: results of the Resistance and Endurance exercise After ChemoTherapy (REACT) study. BMC Medicine, 13, 275. https://doi.org/10.1186/s12916-015-0513-2

Kessels, E., Husson O., \& Van der Feltz-Cornelis, C. M. (2018). The effect of exercise on cancer-related fatigue in cancer survivors: a systematic review and meta-analysis. Neuropsychiatric Diseas and Treatment, 14, 479-494. https://doi.org/10.2147/ndt.s150464

Lane, R. J., Barrett, M. C., Taylor, D. J., Kemp, G. J., \& Lodi, R. (1998). Heterogeneity in chronic fatigue syndrome: Evidence from magnetic resonance spectroscopy of muscle. Neuromuscular Disorders, 8(3-4), 204-209. https://doi.org/10.1016/s0960-8966(98)00021-2

Lee, J. Q., Simmonds, M. J., Wang, S., \& Novy, D. M. (2003). Differences in physical performance between men and women with and without lymphoma. Archive of Physical Medicine Rehabilitation, 84(12), 1747-1752.

Liberati, A., Altman, D. G., Tetzlaff, J., Mulrow, C., Gøtzsche, P. C., loannidis, J. P., Clarke, M., Devereaux, P. J., Kleijnen, J., \& Moher, D. (2009). The PRISMA Statement for Reporting Systematic Reviews and Meta-Analyses of Studies That Evaluate Health Care Interventions: Explanation and Elaboration. PLOS MED, 6(7), e1000100. https://doi.org/10.1371/journal.pmed.1000100

McCully, K. K., Natelson, B. H., Lotti, S., Sisto, S., \& Leigh, J. S. (1996). Reduced oxidative muscle metabolism in chronic fatigue syndrome. Muscle \& Nerve, 19(5), 621-625. https://doi.org/10.1002/(sici)1097-4598(199605)19:5<621::aid-mus10>3.0.co;2-q

Mendes, M. A., da Silva, I., Ramires, V., Reichert, F., Martins, R., Ferreira, R., \& Tomasi, E. (2018). Metabolic equivalent of task (METs) thresholds as an indicator of physical activity intensity. PLOS ONE, 13(7), e0200701. https://doi.org/10.1371/journal.pone.0200701 
Mendoza, T. R., Wang, X. S., Cleeland, C. S., Morrissey, M., Johnson, B. A., Wendt, J. K., \& Huber, S. L. (1999). The rapid assessment of fatigue severity in cancer patients: use of the Brief Fatigue Inventory. $\quad$ Cancer, $\quad 85(5), \quad$ 1186-1196. $\quad$ https://doi.org/10.1002/(sici)10970142(19990301)85:5<1186::aid-cncr24>3.0.c0;2-n

Midtgaard, J., Baadsgaard, M. T., Rasmussen, B., Quist, M., Andersen, C., Rørth, M., \& Adamsen, L. (2009). Self-reported physical activity behaviour; exercise motivation and information among Danish adult cancer patients undergoing chemotherapy. European Journal of Oncology Nursing, 13(2), 116121. https://doi.org/10.1016/.ejon.2009.01.006

Miller, S. A. (2001). Four Elements of the Clinical Question: PICO worksheet and search strategy.

Moher, D., Liberati, A., Tezlaff, J., \& Altman, D. G. (2009). Preferred Reporting Items for Systematic Reviews and Meta-Analyses: The PRISMA Statement. PLOS MED, 6(7), e1000097. https://doi.org/10.1371/journal.pmed.1000097

Monga, U., Garber, S. L., Thornby, J., Vallbona, C., Kerrigan, A. J., Monga, T. N., \& Zimmermann, K. P. (2007). Exercise prevents fatigue and improves quality of life in prostate cancer patients undergoing radiotherapy. Archives of Physical Medicine and Rehabilitation, 88(11), 1416-1422. https://doi.org/10.1016/i.apmr.2007.08.110

Mustian, K. M., Alfano, C. M., Heckler, C., Kleckner, A. S., Kleckner, I. R., Leach, C. R., Mohr, D., Palesh, O. G., Peppone, L. J., Piper, B. F., Scarpato, J., Smith, T., Sprod, L. K., Miller, \& S. M. (2017). Comparison of Pharmaceutical, Psychological, and Exercise Treatments for Cancer-Related Fatigue: A Meta-analysis. JAMA Oncology, 3(7), 961-968. https://doi.org/10.1001/jamaoncol.2016.6914

National Comprehensive Cancer Network. (2000). NCCN Practice Guidelines for Cancer-Related Fatigue. Oncology, 14(11A), 151-161.

National Comprehensive Cancer Network (2003). National Comprehensive Cancer Network practice guidelines cancer-related fatigue panel 2003 guidelines. Journal of National Comprehensive Cancer Network, 1(3), 308. https://doi.org/10.6004/inccn.2003.0029

National comprehensive cancer network (2015). Cancer-Related Fatigue, Version 2.2015: Clinical Practice Guidelines in Oncology. Journal of the National Comprehensive Cancer Network, 13(8), 1012-1039. https://doi.org/10.6004/inccn.2015.0122

Noonan, V., \& Dean, E. (2000). Submaximal exercise testing: clinical application and interpretation. Physical Therapy, 80(8), 782-807. https://doi.org/10.1093/pti/80.8.782

Parkin, D. M., Boyd, L., \& Walker, L. C. (2011). The fraction of cancer attributable to lifestyle and environmental factors in the UK in 2010. British Journal of Cancer, 105(suppl2), 77-81.

Paulo, T. R., Rossi, F. E., Viezel, J., Tosello, G. T., Seidinger, S. C., Simões, R. R., de Freitas, R., \& Freitas, I. F. (2019). The impact of an exercise program on quality of life in older breast cancer survivors undergoing aromatase inhibitor therapy: a randomized controlled trial. Health and Quality of Life Outcomes, 17(1), 17. https://doi.org/10.1186/s12955-019-1090-4

Penttinen, H., Utriainen, M., Kellokumpu-Lehtinen, P. L., Raitanen, J., Sievänen, H., Nikander, R., Blomqvist, C., Huovinen, R., Vehmanen, L., \& Saarto, T. (2019). Effectiveness of a 12-month Exercise Intervention on Physical Activity and Quality of Life of Breast Cancer Survivors; Five-year Results of the BREX-study. In Vivo, 33(3), 881-888. https://doi.org/10.21873/invivo.11554

Pinto, B. M., Frierson, G. M., Rabin, C., Trunzo, J. J., \& Marcus, B. H. (2005). Home-based physical activity intervention for breast cancer patients. Journal of Clinical Oncology, 23(15), 3577-3587. https://doi.org/10.1200/jco.2005.03.080

Piper, B. (1997). Measuring fatigue. In M. Frank-Stromborg, S. J. Olsen, \& J. \& Barlett (Ed.), Instruments for clinical health-care research (pp. 482-496). Sudbury: Jones \& Barlett publishers. 
Piper, B. F., Dibble, S. L., Dodd, M. J., Weiss, M. C, Slaughter, R. E., \& Paul, S. M. (1998). The revised Piper Fatigue Scale: psychometric evaluation in women with breast cancer. Oncology Nursing Forum, 25(4), 677-684. https://doi.org/10.1037/t18854-000

Piper, B., Lindsey A, Dodd, M., Ferketich, S., Paul, S. M., \& Weller, S. (1989). The development of an instrument to measure the subjective dimension of fatigue. In S. G. Funk, \& S. G. Funk (Ed.), Key aspects of comfort (pp. 199-216). New York: Springer Pubblishing Company.

Robinson, K. D., \& Posner, J. D. (1992). Patterns of self-care need, and interventions related to biologic response modifier therapy: fatigue as a model. Seminars in Oncology Nursing Journal, 8(4 suppl 1), 17-22. https://doi.org/10.1016/0749-2081(92)90050-d

Ryan, J. L., Carroll, J. K., Ryan, E. P., Mustian, K. M., Fiscella, K., \& Morrow, G. R. (2007). Mechanisms of cancer-related fatigue. Oncologist, 12(Suppl 1), 22-34. https://doi.org/10.1634/theoncologist.12$\underline{s 1-22}$

Schmidt, M. E., Wiskemann, J., Armabrust, P., Glassley, P., Stigge, R., Northfelt, D., Mikhael, J., Aguirre, A., Bennett, R. M., \& Mesa, R. A. (2015). Effects of resistance exercise on fatigue and quality of life in breast cancer patients undergoing adjuvant chemotherapy: A randomized controlled trial. International Journal of Cancer, 137(2), 471-480. https://doi.org/10.1002/ijc.29383

Schwart, A., \& Meek, P. (1999). Additional contruct of validity of the Schwartz cancer fatigue scale. Journal of Nursing Measurement, 7(1), 35-45.

Schwartz, A. (1998). The Schwartz Cancer Fatigue Scale: testing reliability and validity. Oncology Nursing Forum, 25(4), 711-717.

Segal, R. J., Reid, R. D., Courneya, K. S., Sigal, R. J., Kenny, G. P., Prud'Homme, D. G., Malone, S. C., Wells, G. A., Scott, C. G., \& Slovinec D'Angelo, M. E. (2009). Randomized controlled trial of resistance or aerobic exercise in men receiving radiation therapy for prostate cancer. Journal of Clinical Oncology, 27(3), 344-351. https://doi.org/10.1200/jco.2007.15.4963

Shobeiri, F., Masoumi, S. Z., Nikravesh, A., Heidari Moghadam, \& R., Karami, M. (2016). The Impact of Aerobic Exercise on Quality of Life in Women with Breast Cancer: A Randomized Controlled Trial. Journal of Research in Health Sciences, 16(3), 127-132.

Sitzia, J., \& Huggins, L. (1998, January- February). Side effects of cyclophosphamide, methotrexate, 5fluorouracil (CMF) chemotherapy for breast cancer. Cancer Practice, 6(1), 13-21. https://doi.org/10.1046/j.1523-5394.1998.1998006013.x

Smets, E. M., Garssen, B., Bonke, B., \& De Haes, J. C. (1995). The multidimensional fatigue inventory (MFI). Psychometric qualities of an instrument to assess fatigue. Journal of Psychosomatic Research, 39(3), 315-325. https://doi.org/10.1016/0022-3999(94)00125-0

Stefani, L., Pedrizzetti, G., \& Galanti, G. (2016). Clinical application of 2D speckle tracking strain for assessing cardiotoxicity in oncology. Journal of Functional Morphology and Kinesiology, 1(4), 343354. https://doi.org/10.3390/jfmk1040343

Steindorf, K., Schmidt, M. E., Klassen, O., Ulrich, C. M., Oelmann, J., Habermann, N., Beckhove, P., Owen, R., Debus, J., Wiskemann, J., \& Potthoff, K. (2014). Randomized, controlled trial of resistance training in breast cancer patients receiving adjuvant radiotherapy: results on cancer-related fatigue and quality of life. Annals of Oncology, 25(11), 2237-2243. https://doi.org/10.1093/annonc/mdu374

Stone, P., Hardy, J., Huddart, R., A'Hern, R., \& Richards, M. (2000). Fatigue in patients with prostate cancer receiving hormone therapy. European Journal of Cancer, 36(9), 1134-1141. https://doi.org/10.1016/s0959-8049(00)00084-8

Stout, N. L., Baima, J., Swisher, A. K., Winters-Stone, K. M., \& Welsh, J. (2017). A Systematic Review of Exercise Systematic Reviews in the Cancer Literature (2005-2017). PM\&R, 28(5), S347-S384. https://doi.org/10.1016/j.pmri.2017.07.074 
Sutherland, H. J., Walker, P., \& Till, J. E. (1999). The development of a method for determining oncology patients' emotional distress using linear analogue scales. Cancer Nursing, 11(5), 303-308. https://doi.org/10.1097/00002820-198810000-00006

Torre, L., Siegel, R., \& Jemal, A. (2015). Global Cancer Facts \& Figs. American Cancer Society, 65(2), 87-108.

Van Waart, H., Stuiverm, M. M., van Harten, W. H., Geleijn, E., Kieffer, J. M., Buffart, L. M., de MaakerBerkhof, M., Boven, E., Schrama, J., Geenen, M. M., Meerum Terwogt, J. M., van Bochove, A., Lustig, V., van den Heiligenberg, S. M., Smorenburg, C. H., Hellendoorn-van Vreeswijk, J. A., Sonke, G. S., \& Aaronson, N. K. (2015). Effect of low-intensity physical activity and moderate-To highintensity physical exercise during adjuvant chemotherapy on physical fitness, fatigue, and chemotherapy completion rates: Results of the PACES randomized clinical trial. Journal of Clinical Oncology, 33(17), 1918-1927. https://doi.org/10.1200/jco.2014.59.1081

Wasserstein, R. L., \& Lazar, N. A. (2016). The ASA's Statement on p-Values: Context, Process, and $\begin{array}{llll}\text { Purpose. The American } & \text { Statistician, } & \text { 70(2), } & \text { 133. }\end{array}$ https://doi.org/10.1080/00031305.2016.1154108 